\title{
How to harvest free fibula flap for mandibular reconstruction? Practical tips and tricks
}

\author{
Chirom A Singh, Rajeev Kumar and Pirabu Sakthivel* \\ Department of Otorhinolaryngology \& Head and Neck surgery, All India Institute of Medical Sciences, New Delhi, India
}

\begin{abstract}
The initial use of free fibular flap was limited to the reconstruction of long bone defects of the extremities until 1989, where this flap was used for restoration of segmental mandibulectomy defects. Since then a number of detailed anatomic dissections and publications have helped to clarify the surgical anatomy of this versatile flap. The primary purpose of this video is to describe each and every step along with our easy tips for harvesting this versatile flap for primary mandibular reconstruction.
\end{abstract}

\section{Introduction}

The fibula is a long non-weight bearing bone of the lower extremity with adequate cortical thickness rendering it one of the strongest bones available for mandibular reconstruction [1]. Approximately 22 to 25 $\mathrm{cm}$ of bone can be harvested while preserving 6 to $7 \mathrm{~cm}$ of bone both distally and proximally to maintain the integrity of the ankle and knee joints as well as the common peroneal nerve, making this donor site unique in terms of the ability to restore total or subtotal defects of the mandible [2]. The strength of the cortical bone effectively withstands the powerful forces of mastication when used for mandibular reconstruction. The narrow fibula is also preferred in the secondary reconstruction of mandibular defects that involve the ramus and condyle as it is much easier to pass through the small tunnel for the placement of the graft into the glenoid fossa. The long straight fibula can be contoured to match the shape of mandible by creating wedge shaped osteotomies without compromising distal bone circulation. The thick cortical bone of the fibula appears to accept dental implants well $[3,4]$.

\section{Fibular flap anatomic considerations}

The fibula can be transferred either as a free osseous or as a free osteocutaneous flap based on septocutaneous or musculocutaneous perforators arising from the peroneal artery and vein along the posterior crural septum. The vascular pedicle to the fibular flap although consistent in the location and calibre of the vessel's lumen is often limited in length by the bifurcation of the posterior tibial artery [4].

The peroneal artery and vein run along the entire length of the fibula without a significant change in the calibre of the vessel. This arrangement permits the use of the fibula as a "flow-through" flap to supply a second free flap anastomosed to the free ends of the distal artery and vein.

When reconstructing the mandible, the bone should be oriented so that the vascular pedicle of the fibula IS located on the lingual surface of the neomandible, which places the skin paddle along the inferior border. The skin can be delivered into the oral cavity by transposing it over the buccal surface, which also provides cove rage of the fixation hardware. In addition, the cuff of the flexor hallucis longus provides augmentation in the submandibular region [4].

The lateral sural cutaneous nerve can be harvested and anastomosed to a suitable recipient nerve to restore sensation to the skin component and this can also be used as a sensate osteocutaneous flap [5].

How to harvest Free Fibula flap for Mandibular reconstructionPractical tips and tricks
Department of Otorhinolaryngology and HeadNeck Surgery, All India Institute of Medical Sciences, New Delhi.

Dr. Rajeev Kumar

Dr. Pirabu Sakthivel

Harvesting free fibula flap for mandibular reconstruction

*Correspondence to: Pirabu Sakthivel, Room no 4057, ENT office, Fourth floor, Department of ENT \& Head and Neck surgery, All India Institute of Medical Sciences, New Delhi - 110029, India, Tel: 9958744547; E- mail: pirabusakthivel@gmail.com

Received: March 16, 2019; Accepted: March 25, 2019; Published: March 29, 2019 


\section{References}

1. Serra JM, Paloma V, Mesa F, Ballesteros A (1991) The vascularized fibula graft in mandibular reconstruction. J Oral Maxillofac Surg 49: 244-250. [Crossref]

2. Brown JS, Lowe D, Kanatas A, Schache A (2017) Mandibular reconstruction with vascularised bone flaps: a systematic review over 25 years. Br J Oral Maxillofac Surg 55: 113-126. [Crossref]
3. Lonie S, Herle P, Paddle A, Pradhan N, Birch T, et al. (2016) Mandibular reconstruction: meta-analysis of iliac- versus fibula-free flaps. ANZ J Surg 86: 337-342. [Crossref]

4. Abou-Foul AK, Fasanmade A, Prabhu S, Borumandi F (2017) Anatomy of the vasculature of the lower leg and harvest of a fibular flap: a systematic review. Br J Oral Maxillofac Surg 55: 904-910. [Crossref]

5. Boyd JB, Caton AM, Mulholland RS, Tong L, Granzow JW (2013) The sensate fibula osteocutaneous flap: neurosomal anatomy.JPlast Reconstr Aesthet Surg 66: 1688-94. [Crossref]

Copyright: $\left({ }^{2} 2019\right.$ Singh CA. This is an open-access article distributed under the terms of the Creative Commons Attribution License, which permits unrestricted use, distribution, and reproduction in any medium, provided the original author and source are credited. 